\title{
Not All Cases of Visual Snows are Benign: Mimics of Visual Snow Syndrome
}

\author{
Chenyue Hang' \\ Lakshmi Leishangthem ${ }^{2}$ \\ Yan Yan (iD ${ }^{3}$ \\ 'Ottawa-Shanghai Joint School of \\ Medicine, Shanghai Jiao Tong University \\ School of Medicine, Shanghai, People's \\ Republic of China; ${ }^{2}$ Department of \\ Neurology and Ophthalmology, \\ University of Connecticut, Farmington, \\ CT, USA; ${ }^{3}$ Department of \\ Ophthalmology, Ren Ji Hospital, Shanghai \\ Jiao Tong University School of Medicine, \\ Shanghai, People's Republic of China
}

Correspondence: Yan Yan Department of Ophthalmology, Ren Ji Hospital, Shanghai Jiao Tong University School of Medicine, Pujian Road 160, Shanghai, People's Republic of China Tel +8621 58752345

Email hz2004yan@gmail.com

\begin{abstract}
Visual snow syndrome (VSS) is a clinical disorder characterized by pan-field visual disturbance. It is a diagnosis of exclusion since its pathophysiology remains unknown. Excluding other mimics is of great significance since some serious pathologies can have secondary visual snow (VS) as an initial presentation. Delayed or incorrect diagnosis of these VSS mimics may lead to permanent vision loss or even death. The purpose of this review is to help physicians distinguish VSS mimics promptly to avoid bad outcomes. The authors performed a PubMed literature search of articles, case reports, and reviews describing VS symptoms in patients with underlying diseases other than VSS. The red flags of secondary VS symptoms were highlighted, such as new-onset or intermittent VS, unilateral or quadrant VS, and accompanied ocular or neurological deficits. There are four main categories of VSS mimics, ie, including neurological disorders, ocular pathologies, drug-related VS, and other systemic diseases. The physicians could largely exclude most etiologies based on history taking, ophthalmologic and neurologic examinations, and neuroimaging. Further research in VS should carefully define and unify the inclusion and exclusion criteria of this disorder and investigate these secondary VS conditions and their pathogenesis.
\end{abstract}

Keywords: birdshot chorioretinopathy, Creutzfeldt-Jakob disease, hallucinogen persisting perception disorder, migraine with visual aura, visual snow

\section{Introduction}

Visual snow (VS) describes a phenomenon where patients see numerous flickering dots throughout the visual field, often described as the static look of an untuned television. It is commonly noticeable on a plain background but more attenuated on a textured background. ${ }^{1-4}$ Liu et al first reported it in 3 of 10 migraineurs in $1995 .^{2}$ In 2005, Jager et al first used the term "visual snow phenomenon" to describe this situation, ${ }^{5}$ while in 2008 and 2011, Wang et $\mathrm{al}^{6}$ and Chen et $\mathrm{al}^{7}$ used the term "persistent visual aura without infarction" to describe it. In 2013, Simpson et al ${ }^{8}$ reported a girl with "positive persistent visual symptoms/visual snow".

The concept of visual snow syndrome (VSS) was raised as a clinically independent syndrome by Schankin et al in $2014 .^{1}$ The estimated prevalence of VSS in the UK is around $2 \% .{ }^{9}$ By definition, the patients with VSS experience at least 3 months of persistent VS and at least 2 additional visual symptoms, such as palinopsia, enhanced entoptic phenomena, photophobia, and impaired night vision. Moreover, the symptoms should not be suggestive of other diseases or symptoms. Metzler et al proposed a modified edition of diagnostic criteria in 2018, replacing "enhanced entopic phenomena" with "other persistent positive visual phenomena". ${ }^{10}$ Figure 1 lists the evolution of terms to describe VS and VSS so far, and some recent interesting research about them. 


\section{CLINICAL DESCRIPTIONS}

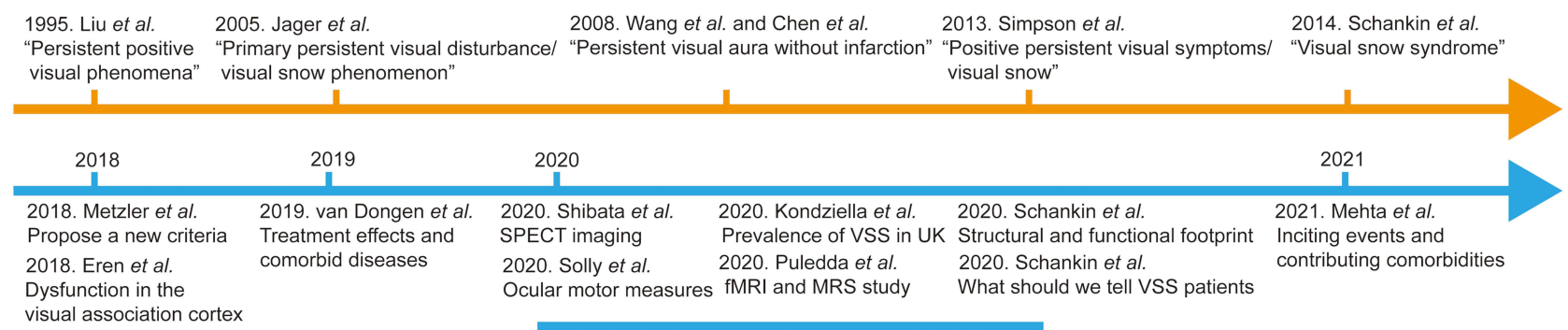

\section{RECENT RESEARCH}

Figure I The evolution of clinical descriptions and recent research of visual snow.

Abbreviations: fMRI, functional magnetic resonance imaging; MRS, magnetic resonance spectroscopy; SPECT, single-photon emission computed tomography; VSS, visual snow syndrome.

There are several theories suggested explaining this visual phenomenon. Visual cortex hyperexcitability presenting as reduced habituation, ${ }^{11}$ and dysfunctional cortical visual processing in $\mathrm{VS}^{12}$ have been demonstrated in electrophysiologic studies. Because color filters in the blue-yellow spectrum reduced VS in some patients probably by regulating the konio-and parvo/magnocellular pathway, thalamocortical dysrhythmia of the visual pathway is thought to be involved in VS. ${ }^{13}$ Finally, unimodal stochastic resonance (SR) in the visual system improves the signal-to-noise ratio and enables people to perceive a subthreshold visual stimulus, and cross-model of SR in the sensory system contributes to VS occurring with tinnitus and migraine. ${ }^{10}$ Multiple ways like single-photon emission computed tomography imaging, ${ }^{14}$ ocular motor measures, ${ }^{15}$ functional magnetic resonance imaging (fMRI), and magnetic resonance spectroscopy (MRS) study $^{16}$ have been used recently to explore VSS. Although the exact pathophysiology of VSS remains mysterious, some treatments have been tried and are effective. ${ }^{17}$

In this review, we defined VSS mimics as those conditions presenting with VS symptoms due to other secondary causes. This condition is also named secondary VS in some papers. For example, Mehta et al reported 89 patients with secondary VS due to an inciting event or attributed to underlying causes in a case series of $248 \mathrm{VS}$ patients. ${ }^{18}$ Clinicians should look out for these secondary causes. Accurate diagnosis is essential to ensure that patients receive appropriate treatment and prognostic information. Delayed or incorrect diagnosis of a secondary VS due to underlying conditions may result in the progression of underlying diseases, leading to permanent vision loss or even death. The purpose of this review is to highlight other diseases presenting with VS symptoms and to ensure that clinicians are aware of other significant disorders that need to be excluded first.

\section{Methods}

We performed a PubMed literature search of available original papers, case reports, and reviews on "visual snow" or "visual snow syndrome" from 1995 to June 2021. Here, the authors discussed the differential diagnosis of VS and secondary VS with underlying causes. In addition, the cases of VSS mimics in the literature were described, categorized, and further discussed in this review. VSS mimics usually result in VS from the underlying diseases and present with or without additional visual disturbances. The presence of these mimics may lead to severe consequences, which makes ruling out other possible etiologies in VS extremely important. The conditions that cause VS symptoms can be categorized into neurological disorders, ocular diseases, drug-related conditions, and systemic diseases. Some common conditions that may result in secondary VS are discussed in detail below.

\section{Results}

\section{Neurological Diseases}

Any neurological condition that affects the occipital visual area might trigger VS, including stroke, epilepsy, multiple sclerosis, neoplastic diseases, degenerative diseases like posterior cortical atrophy, and idiopathic intracranial hypertension (IIH). ${ }^{18}$ 


\section{Migraine with Visual Aura}

VS was believed to be part of visual aura, which Schankin et al opposed. ${ }^{1}$ The distinction of VSS from migraine with aura mainly depends on clinical features. VSS affects the whole visual field, shows no evidence of cortical spreading depression-like mechanism, ${ }^{19}$ lacks classic migraine features like fortification spectra and scotomas, ${ }^{20}$ and cannot be treated effectively with antimigraine drugs. ${ }^{8}$

However, there is no denying that migraine is still a prevalent comorbidity of VSS. A hypothesis for this phenomenon is that VSS and migraine aura both relate to cortical hyperexcitability. ${ }^{21}$ Unal-Cevik et al showed that a young female with VS who also had migraine with aura had left occipital bending on MRI, and her VS symptoms resolved with lamotrigine. ${ }^{22}$ An uncommon type of VS, episodic VS (eVS), happening intermittently (unlike continuously as in most VSS patients), links with migraine attacks and does not present with other additional visual symptoms in VSS. ${ }^{1}$ Different from the common VSS, eVS meets the rhythm of migraine. It occurs only in less than $0.2 \%$ of migraineurs and was described in detail by Hodak et al. ${ }^{19}$

\section{Occipital Epilepsy}

Occipital lobe epilepsy causes paroxysmal visual manifestations during seizures originating from the visual cortex. Typically, the visual disturbance presents as the small, multicolored circles moving horizontally for seconds to minutes. ${ }^{23}$ Polster et al reported that two adolescents with suspected epilepsy fulfilled the diagnostic criteria for VSS. They both had pathological EEG findings of epileptiform activity with photic stimulation with asymmetric amplitudes. ${ }^{24}$

\section{Occipital Stroke}

Catarci described a 74-year-old male with a history of VSS in the past 40 years, who experienced temporary VS symptoms in the upper left visual field, secondary to a right ischemic occipital stroke. Visual field testing showed a corresponding superior left homonymous quadrantanopia. Catarci hypothesized that the stroke resulted in a localized disruption leading to a temporary imbalance in inhibitory circuits of vision. ${ }^{25}$

\section{Creutzfeldt-Jakob Disease}

Creutzfeldt-Jakob disease (CJD) is a rapidly progressive, invariably fatal degeneration of the central nervous system due to prion disease and commonly presents at 50-70 years of age. The main symptoms of CJD include rapidly progressive dementia and associated myoclonus. However, a subtype of sporadic CJD (sCJD) named "the Heidenhain variants of sCJD" has isolated visual symptoms, including blurred vision, visual field loss, and visual processing issues before the deterioration of dementia. ${ }^{26}$ Chen et al reported a 55-year-old man with 15 months of slowly progressive VS, illusory visual motion, and impaired night vision, who was finally diagnosed as SCJD on postmortem examination. ${ }^{27}$ They hypothesized that the pathologic process of CJD in the occipital cortex caused an imbalance between cortical inhibition and excitation, resulting in VS. The existence of other visual symptoms in SCJD may conceal the presence of VS. Abnormalities on neuroimaging are crucial to differentiate VS due to sCJD from VSS in this rare condition.

\section{Glycine Receptor Antibody Syndrome}

Glycine receptor alpha-1 subunit $\left(\mathrm{GlyR}_{\alpha} 1\right)$ is one of the autoantibodies associated with stiff-person spectrum disorder (SPSD). Piquet et al reported that 10 of 17 patients with glycine receptor antibody syndrome had uncommon persistence of visual disturbance, including VS, spiderweb-like images forming shapes, palinopsia, and other visual symptoms. ${ }^{28}$ They described that a 41 -year-old female had visual symptoms of VS, palinopsia, photophobia, visual hallucinations, and whole-brain atrophy on MRI several years preceding stiffness and spasms of the lower extremities. Another 45-year-old female patient presented with visual symptoms had profound visual field constriction and an abnormal electroretinogram. Since $\mathrm{GlyR}_{\alpha} 1$ is an inhibitory neurotransmitter of the human retina, they speculated that GlyR autoimmunity caused dysregulated signaling causing positive visual phenomena, and visual disturbance might be an early sign of the syndrome. ${ }^{28}$

\section{Head Trauma}

Head trauma-related VS may immediately occur after the injury or after 2-12 weeks. ${ }^{18}$ Mehta et al reported 15 cases of VS associated with a head injury, accompanied by postconcussive symptoms and headaches. ${ }^{18}$ Metzler et al presented a 17-year-old boy with persistent VS throughout the visual field after a concussion, who also had separated episodes of headaches. ${ }^{10}$ A young man described by Liu et al experienced VS accompanied by headache after the head and neck trauma. ${ }^{2}$ The possibility of VS after a head injury may relate to the injury to the visual pathways. 
Headaches were responsive to treatment, while VS was commonly unresponsive. ${ }^{2,10}$

\section{Ocular Abnormalities}

Mehta et al reported 7 out of 98 patients with secondary VS had ocular abnormalities, including macular atrophy, central serous retinopathy, vitreous detachment, and multifocal choroiditis with a macular lesion as well as optic atrophy. ${ }^{18}$

\section{Retinal Disease}

In a report of 28 patients with VS by Yoo et al, eight patients with rod-cone dystrophy had VS. ${ }^{43}$ They presented a typical case of rod-cone dystrophy misdiagnosed as VSS. A 36-yearold female with migraine without aura experienced a 6-month history of VS in her entire visual field and additional visual symptoms. She had normal visual acuity, normal fundus, and binasal visual field defect. On electroretinography, she had severely reduced scotopic responses and relatively preserved photopic responses. Bittner demonstrated that decreased central vision correlated with increased awareness of photopsia in retinitis pigmentosa (RP). VS was present in $22 \%$ of patients with $\mathrm{RP}^{29}$ It is speculated that photopsia in RP results from spontaneous discharges from the remodeling inner retina or release phenomena due to lack of input. ${ }^{29}$ The clinicians should provide a thorough ophthalmic examination and an electroretinogram to confirm the diagnosis.

\section{Uveitis}

Posterior uveitis may present with VS as the first manifestation. VS can also start during steroid treatment in multifocal choroiditis with a macular lesion. From a treatment perspective, Adalimumab has been shown to help photopsia and vision but did not show any effect on VS. ${ }^{18}$ Birdshot chorioretinopathy (BSCR) is a posterior chronic bilateral autoimmune uveitis strongly associated with HLA-A29. ${ }^{30}$ In the early stage of BSCR, patients may have symptoms of VS. Patel et al reported that a patient with flickering visual disturbances and previous history of migraines was misdiagnosed as VS. ${ }^{31}$ The following abnormal retinal exam, visual field, and electroretinogram (ERG) differentiated it from VSS and confirmed the diagnosis of BSCR.

\section{Drug-Related VS}

Drug-related VS include hallucinogen persisting perception disorder (HPPD) and the effects of other medicines such as proton pump inhibitor, imiquimod, ciprofloxacin, tamoxifen, amantadine, testosterone, escitalopram, duloxetine, and bupropion as well as IV steroids. ${ }^{18}$

\section{Hallucinogen Persisting Perception Disorder (HPPD)}

HPPD is reexperiencing a perceptual symptom similar to being intoxicated with a hallucinogen during a drug-free state. The common substances causing HPPD include lysergic acid diethylamide (LSD), cannabis, synthetic cannabinoids, phencyclidine, ecstasy, psilocybin, mescaline, and cocaine. ${ }^{18,32}$ In type 2 HPPD, which shows chronic and constant visual symptoms and appears in $0.002 \%$ of hallucinogen users, patients could have symptoms of VS, palinopsia, halos, and after-images. ${ }^{33}$ Eren et al reported that a 31-year-old female with episodic migraine showed symptoms of VS after taking $20 \mathrm{mg}$ citalopram for two weeks, and the VS symptoms persisted after stopping the drug. The ophthalmic and neurologic testing, including brain MRI, was unremarkable. The Liverpool adverse drug reaction causality assessment tool showed a possible adverse drug reaction. ${ }^{34}$ Schatten et al described a 24-year-old female with permanent VS-like flickering in front of both eyes for 1.5 years after consuming a cocktail of amphetamines, hallucinogens, and alcohol. A thorough examination revealed no abnormality, and she was diagnosed with type 2 HPPD. ${ }^{35}$

The reason why the symptoms of HPPD and VSS overlap may lie in the pathophysiology of both of them involving changes in serotonergic synaptic transmission, ${ }^{36,37}$ especially $5 \mathrm{HT}_{2 \mathrm{a}}$-receptors. ${ }^{34}$ However, van Dongen et al suggested that there is still a difference in their pathogenesis since none of the people with VS symptoms after using hallucinogen had a migraine. In the control group, over half of VS patients without the use of hallucinogen had a migraine. ${ }^{38}$ Zobor reported an HPPD patient with abnormal electrooculography and electrically evoked phosphene thresholds, demonstrating the dysfunction of the retina and retinal pigmental epithelium. The causes of visual symptoms could be attributed to both retina and cerebrum. ${ }^{39}$ There are currently no established therapy recommendations for HPPD. There have been case reports of attempts with presynaptic $\alpha 2$-agonists, benzodiazepines, anticonvulsants, and low-dose first-generation antipsychotics. ${ }^{32}$

\section{Other Systemic Diseases}

Despite the diseases mentioned above, there are still many VSS mimics, which have been described in detail in the study 
by Mehta et al, such as immune diseases, infectious diseases, hormone-related conditions, and psychiatric diseases. ${ }^{18}$ Based on this study, VS developed after inciting events will respond better to treatment than occurring spontaneously. Consequently, we need to identify the inciting events and exclude other mimickers before diagnosing VSS. We conclude the inciting factors or causes of secondary VS in Table 1 and handy algorithm for patients with VS in Figure 2.

\section{Discussion}

In this review, we did a literature review and discussed the mimics of VSS, and we recommended the clinical approach to emphasize the exclusion of other significant disorders in the diagnosis of VSS. To diagnose VSS, VS must be lasting for at least 3 months, present with at least 2 other visual symptoms, and cannot be associated with any other diseases. Generally, the red flag symptoms of VS include new-onset VS, onset at an older age, intermittent or sudden exacerbation of VS, quadrant or hemifield VS, unilateral VS, no additional visual disturbance, history of recently discontinued illicit drugs or intake of any medications, accompanied or lateonset neurologic deficit, vision change (visual acuity, color vision, or visual field). Any of the above warning symptoms presented in the patients should alert the clinicians to perform more extensive examinations to rule out serious diseases.
To summarize, there are four main categories of VSS mimics: neurological disorders, ocular pathologies, drugrelated VS, and other systemic diseases. All of them affect a part of the visual pathway and could influence the visual fields. Neurological diseases like SCJD and migraine may trigger VS by affecting the occipital cortex and leading to its hyperexcitability, ${ }^{21,27}$ while drugs may excite the serotonergic synaptic transmission in the same way as VSS. ${ }^{34,37}$ The reasons for retinal and choroidal diseases to cause VS is not fully discussed in the past articles but may relate with dysfunction of visual stimulus transmitting to the brain or abnormal spontaneous discharge of the retinal cells. ${ }^{29}$ Owing to the multiple diseases that may cause VS, it is important for physicians to exclude mimics before diagnosing VSS. Based on history taking, neurologic examination, and neuroimaging, we can largely exclude neurological etiologies. Ocular pathologies can be ruled out by checking the ophthalmologic assessments, including careful fundus examination, ocular coherence tomography (OCT), and ERG. Drug-related conditions could be missed without detailed anamneses.

There are very few effective treatments for VS. Bou Ghannam et al recommended lamotrigine and acetazolamide as first-line treatments of VSS. ${ }^{40}$ Secondary VS may have a better prognosis than VSS based on Mehta et al's

Table I Inciting Factors or Causes of Secondary Visual Snow

\begin{tabular}{|c|c|c|c|}
\hline Neurological Diseases & Ocular Diseases & $\begin{array}{l}\text { Drug Associated } \\
\text { Conditions }\end{array}$ & $\begin{array}{l}\text { Systemic Diseases/ } \\
\text { Conditions }\end{array}$ \\
\hline $\begin{array}{l}\text { - Migraine with visual aura } \\
\text { - Occipital epilepsy } \\
\text { - Heidenhain variant of CJD } \\
\text { - Glycine receptor antibody } \\
\text { syndrome } \\
\text { - Stroke } \\
\text { - MS with or without optic neuritis } \\
\text { - IIH } \\
\text { - Head trauma }\end{array}$ & $\begin{array}{l}\text { - Vitreous detachment } \\
\text { - Macular atrophy } \\
\text { - Central serous retinopathy } \\
\text { - Retinitis pigmentosa } \\
\text { - Multifocal choroiditis with macular } \\
\text { lesion } \\
\text { - Birdshot chorioretinopathy } \\
\text { - Optic atrophy }\end{array}$ & $\begin{array}{l}\text { - HPPD } \\
\text { Lysergic acid diethylamide } \\
\text { Cannabis } \\
\text { Synthetic cannabinoids } \\
\text { Phencyclidine } \\
\text { Ecstasy } \\
\text { Psilocybin } \\
\text { Mescaline } \\
\text { - PPI } \\
\text { - Imiquimod } \\
\text { - Ciprofloxacin } \\
\text { - Tamoxifen } \\
\text { - Amantadine } \\
\text { - Testosterone } \\
\text { - Escitalopram } \\
\text { - Duloxetine } \\
\text { - Bupropion } \\
\text { - IV steroids } \\
\text { - OCP }\end{array}$ & $\begin{array}{l}\text { - Post infections } \\
\text { GI illness } \\
\text { Systemic } \\
\text { URI/bronchitis } \\
\text { - Hormone-related } \\
\text { Menarche } \\
\text { Pregnancy } \\
\text { - APS } \\
\text { - Severe hypothyroidism } \\
\text { - Malignant hypertension } \\
\text { - Psychologic diseases }\end{array}$ \\
\hline
\end{tabular}

Abbreviations: APS, antiphospholipid antibody syndrome; CJD, Creutzfeldt-Jakob Disease; HPPD, hallucinogen persisting perception disorder; IIH, idiopathic intracranial hypertension; GI, gastrointestinal; IV, intravenous; MS, multiple sclerosis; OCP, oral contraceptive; PPI, proton pump inhibitor; URI, upper respiratory infection. 


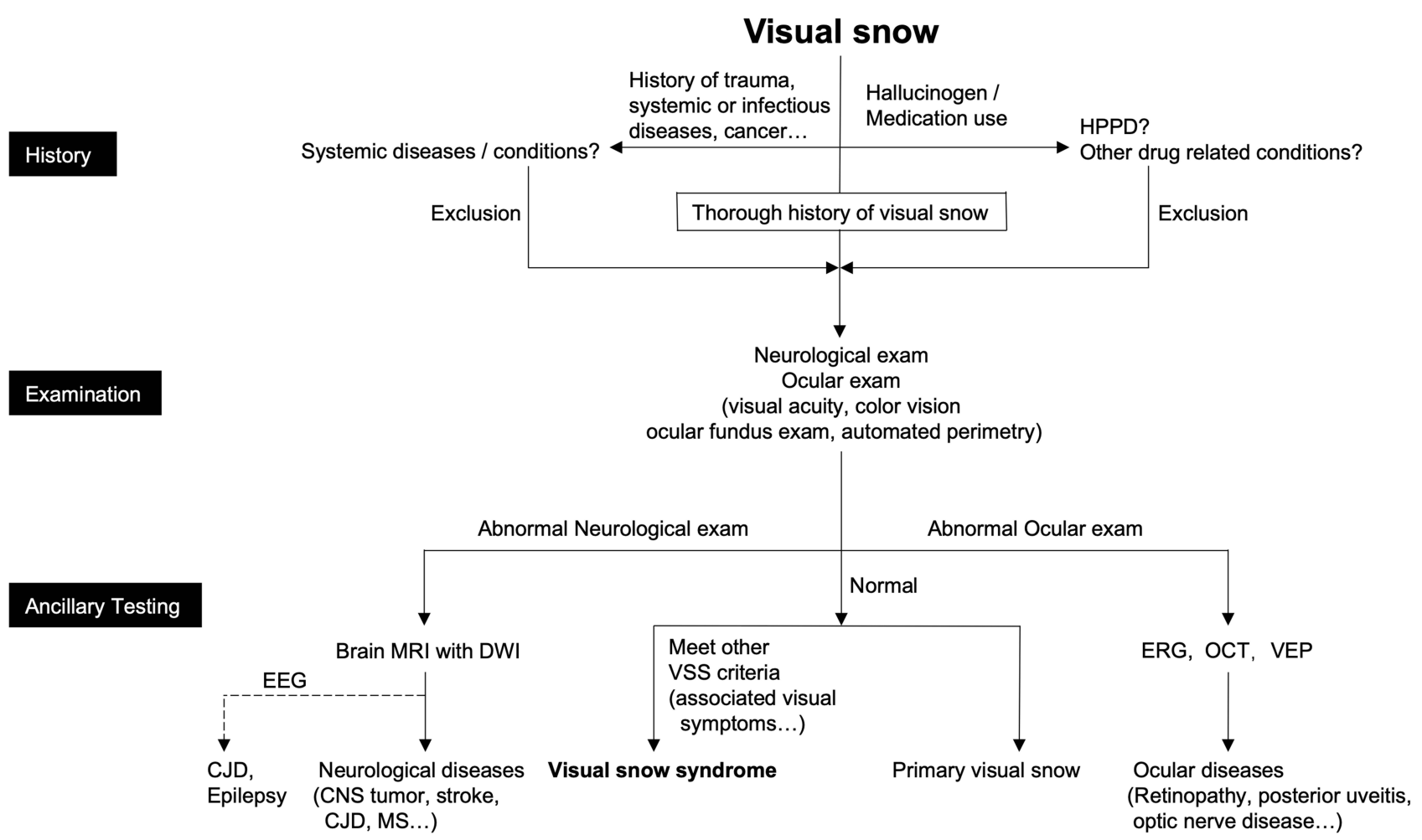

Figure 2 Clinical algorithm for patients with visual snow.

Abbreviations: CNS, central nervous system; CJD, Creutzfeldt-Jakob disease; DWI, diffusion-weighted imaging; EEG, electroencephalogram; ERG, electroretinogram; HPPD, hallucinogen persisting perception disorder; MRI, magnetic resonance imaging; MS, multiple sclerosis; OCT, optical coherence tomography; VEP, visual evoked potentials; VSS, visual snow syndrome.

study. ${ }^{18}$ In the treatment of the primary diseases, secondary VS in some cases subsided partially or entirely, such as brain tumor, post-infection, IIH, and HPPD. ${ }^{18}$ Lamotrigine, topiramate, lorazepam, clonazepam, amitriptyline, acetazolamide worked well in some cases. ${ }^{18}$ In some neurodegenerative and ocular diseases, VS was refractory to any treatment. ${ }^{18}$ Color filters of the yellowblue color spectrum ${ }^{41}$ and repetitive transcranial magnetic stimulation of the visual cortex ${ }^{42}$ are also helpful in some patients with VSS. These strategies can be used to treat secondary VS in refractory cases.

\section{Conclusion}

The concept of visual snow was first introduced 16 years ago. Although the clinicians and researchers in this field widely accepted the definition of VSS, there are still many mysteries about the pathophysiology and effective treatment of this entity. Meanwhile, the conditions with VS but not VSS should be given extreme attention in the clinic. The delayed or incorrect diagnosis of underlying diseases will result in permanent vision loss or even death. Detailed anamneses and appropriate investigations such as neuroimaging and ophthalmological examinations are very helpful in ruling out these conditions.
Further studies of visual snow should also focus on these secondary VS conditions and the investigation of its pathogenesis.

\section{Disclosure}

The authors report no conflicts of interest in this work.

\section{References}

1. Schankin CJ, Maniyar FH, Digre KB, Goadsby PJ. 'Visual snow' a disorder distinct from persistent migraine aura. Brain. 2014;137(Pt 5):1419-1428. doi:10.1093/brain/awu050

2. Liu GT, Schatz NJ, Galetta SL, Volpe NJ, Skobieranda F, Kosmorsky GS. Persistent positive visual phenomena in migraine. Neurology. 1995;45(4):664-668. doi:10.1212/WNL.45.4.664

3. Bessero AC, Plant GT. Should 'visual snow' and persistence of afterimages be recognised as a new visual syndrome? J Neurol Neurosurg Psychiatry. 2014;85(9):1057-1058. doi:10.1136/jnnp-2013-306827

4. Zambrowski O, Ingster-Moati I, Vignal-Clermont $\mathrm{C}$, Robert MP. [The visual snow phenomenon]. J Fr Ophtalmol. 2014;37(9):722-727. French. doi:10.1016/j.jfo.2014.08.001

5. Jäger HR, Giffin NJ, Goadsby PJ. Diffusion- and perfusion-weighted MR imaging in persistent migrainous visual disturbances. Cephalalgia. 2005;25(5):323-332. doi:10.1111/j.1468-2982.2004.00 858.x

6. Wang YF, Fuh JL, Chen WT, Wang SJ. The visual aura rating scale as an outcome predictor for persistent visual aura without infarction. Cephalalgia. 2008;28(12):1298-1304. doi:10.1111/j.1468-2982.2008. 01679.x 
7. Chen WT, Lin YY, Fuh JL, Hämäläinen MS, Ko YC, Wang SJ. Sustained visual cortex hyperexcitability in migraine with persistent visual aura. Brain. 2011;134(Pt 8):2387-2395. doi:10.1093/brain/ awr157

8. Simpson JC, Goadsby PJ, Prabhakar P. Positive persistent visual symptoms (visual snow) presenting as a migraine variant in a 12-year-old girl. Pediatr Neurol. 2013;49(5):361-363. doi:10.1016/ j.pediatrneurol.2013.07.005

9. Kondziella D, Olsen MH, Dreier JP. Prevalence of visual snow syndrome in the UK. Eur J Neurol. 2020;27(5):764-772. doi:10.1111/ene. 14150

10. Metzler AI, Robertson CE. Visual snow syndrome: proposed criteria, clinical implications, and pathophysiology. Curr Neurol Neurosci Rep. 2018;18(8):52. doi:10.1007/s11910-018-0854-2

11. Yildiz FG, Turkyilmaz U, Unal-Cevik I. The clinical characteristics and neurophysiological assessments of the occipital cortex in visual snow syndrome with or without migraine. Headache. 2019;59 (4):484-494. doi:10.1111/head.13494

12. Eren O, Rauschel V, Ruscheweyh R, Straube A, Schankin CJ. Evidence of dysfunction in the visual association cortex in visual snow syndrome. Ann Neurol. 2018;84(6):946-949. doi:10.1002/ana.25372

13. Lauschke JL, Plant GT, Fraser CL. Visual snow: a thalamocortical dysrhythmia of the visual pathway? $J$ Clin Neurosci. 2016;28:123-127. doi:10.1016/j.jocn.2015.12.001

14. Shibata M, Tsutsumi K, Iwabuchi Y, et al. [(123)I]-IMP single-photon emission computed tomography imaging in visual snow syndrome: a case series. Cephalalgia. 2020;40 (14):1671-1675. doi:10.1177/0333102420950454

15. Solly EJ, Clough M, McKendrick AM, Foletta P, White OB, Fielding J. Ocular motor measures of visual processing changes in visual snow syndrome. Neurology. 2020;95(13):e1784-e1791. doi:10.1212/WNL.0000000000010372

16. Puledda F, Ffytche D, Lythgoe DJ, et al. Insular and occipital changes in visual snow syndrome: a BOLD fMRI and MRS study. Ann Clin Transl Neurol. 2020;7(3):296-306. doi:10.1002/acn3.50986

17. van Dongen RM, Waaijer LC, Onderwater GLJ, Ferrari MD, Terwindt GM. Treatment effects and comorbid diseases in 58 patients with visual snow. Neurology. 2019;93(4):e398-e403. doi:10.1212/ WNL.0000000000007825

18. Mehta DG, Garza I, Robertson CE. Two hundred and forty-eight cases of visual snow: a review of potential inciting events and contributing comorbidities. Cephalalgia. 2021;333102421996355. doi:10.1177/0333102421996355

19. Hodak J, Fischer U, Bassetti CLA, Schankin CJ. Episodic visual snow associated with migraine attacks. JAMA Neurol. 2020;77 (3):392-393. doi:10.1001/jamaneurol.2019.4050

20. van Dongen RM, Haan J. Symptoms related to the visual system in migraine. F1000Res. 2019;8:1219. doi:10.12688/f1000research.18768.1

21. Chen WT, Fuh JL, Lu SR, Wang SJ. Persistent migrainous visual phenomena might be responsive to lamotrigine. Headache. 2001;41 (8):823-825. doi:10.1046/j.1526-4610.2001.01150.x

22. Unal-Cevik I, Yildiz FG. Visual snow in migraine with aura: further characterization by brain imaging, electrophysiology, and treatmentCase Report. Headache. 2015;55(10):1436-1441. doi:10.1111/head. 12628

23. Fraser CL, Lueck CJ. Illusions, hallucinations, and visual snow. Handb Clin Neurol. 2021;178:311-335.

24. Polster T, Schaefer S, Panzer A. Visual snow-two adolescents with a new syndrome different from migraine or epilepsy. Neuropediatrics. 2019;50 (S 02):GNP-FV20.

25. Catarci T. Occipital ischaemic stroke after visual snow phenomenon a case report. Cephalalgia. 2021;41(7):871-874. doi:10.1177/0333 102420985444
26. Cooper SA, Murray KL, Heath CA, Will RG, Knight RS. Isolated visual symptoms at onset in sporadic Creutzfeldt-Jakob disease: the clinical phenotype of the "Heidenhain variant". Br J Ophthalmol. 2005;89(10):1341-1342.

27. Chen BS, Lance S, Lallu B, Anderson NE. Visual snow: not so benign. J Clin Neurosci. 2019;64:37-39. doi:10.1016/j.jocn.2019.03.023

28. Piquet AL, Khan M, Warner JEA, et al. Novel clinical features of glycine receptor antibody syndrome: a series of 17 cases. Neurol Neuroimmunol Neuroinflamm. 2019;6(5):e592. doi:10.1212/NXI.00 00000000000592

29. Bittner AK, Diener-West M, Dagnelie G. Characteristics and possible visual consequences of photopsias as vision measures are reduced in retinitis pigmentosa. Invest Ophthalmol Vis Sci. 2011;52(9):63 70-6376. doi:10.1167/iovs.11-7195

30. Minos E, Barry RJ, Southworth S, et al. Birdshot chorioretinopathy: current knowledge and new concepts in pathophysiology, diagnosis, monitoring and treatment. Orphanet J Rare Dis. 2016;11(1):61. doi:10.1186/s13023-016-0429-8

31. Patel RC, Vitale AT, Creel DJ, Digre KB. Not all that flickers is snow. $J$ Neuroophthalmol. 2021;41(1):e97-e99. doi:10.1097/WNO.000 0000000000935

32. Martinotti G, Santacroce R, Pettorruso M, et al. Hallucinogen persisting perception disorder: etiology, clinical features, and therapeutic perspectives. Brain Sci. 2018;8(3):3. doi:10.3390/brainsci8030047

33. Halpern JH, Lerner AG, Passie T. A review of Hallucinogen Persisting Perception Disorder (HPPD) and an exploratory study of subjects claiming symptoms of HPPD. Curr Top Behav Neurosci. 2018;36:333-360.

34. Eren OE, Schöberl F, Schankin CJ, Straube A. Visual snow syndrome after start of citalopram-novel insights into underlying pathophysiology. Eur J Clin Pharmacol. 2021;77(2):271-272. doi:10. 1007/s00228-020-02996-9

35. Schatten H, Eter N, Mihailovic N. [Visual snow in hallucinogen-persisting perception disorder]. Ophthalmologe. 2020;117(11):1112-1115. doi:10.1007/s00347-020-01056-y

36. Halpern JH, Pope HG Jr. Hallucinogen persisting perception disorder: what do we know after 50 years? Drug Alcohol Depend. 2003;69 (2):109-119. doi:10.1016/S0376-8716(02)00306-X

37. Abraham HD, Aldridge AM, Gogia P. The psychopharmacology of hallucinogens. Neuropsychopharmacology. 1996;14(4):285-298. doi:10.1016/0893-133X(95)00136-2

38. van Dongen RM, Alderliefste GJ, Onderwater GLJ, Ferrari MD, Terwindt GM. Migraine prevalence in visual snow with prior illicit drug use (Hallucinogen Persisting Perception Disorder) versus without. Eur J Neurol. 2021. doi:10.1111/ene.14914

39. Zobor D, Strasser T, Zobor G, et al. Ophthalmological assessment of cannabis-induced persisting perception disorder: is there a direct retinal effect? Doc Ophthalmol. 2015;130(2):121-130. doi:10.1007/ s10633-015-9481-2

40. Bou Ghannam A, Pelak VS. Visual snow: a potential cortical hyperexcitability syndrome. Curr Treat Options Neurol. 2017;19(3):9. doi:10.1007/s11940-017-0448-3

41. Fraser CL, White OB. There's something in the air. Surv Ophthalmol. 2019;64(5):729-733. doi:10.1016/j.survophthal.2018.08.004

42. Grey V, Klobusiakova P, Minks E. Can repetitive transcranial magnetic stimulation of the visual cortex ameliorate the state of patients with visual snow? Bratisl Lek Listy. 2020;121(6):395-399.

43. Yoo YJ, Yang HK, Choi JY, Kim JS, Hwang JM. Neuro-ophthalmologic Findings in Visual Snow Syndrome. J Clin Neurol. 2020;16 (4):646-652. doi:10.3988/jcn.2020.16.4.646 


\section{Publish your work in this journal}

Neuropsychiatric Disease and Treatment is an international, peerreviewed journal of clinical therapeutics and pharmacology focusing on concise rapid reporting of clinical or pre-clinical studies on a range of neuropsychiatric and neurological disorders. This journal is indexed on PubMed Central, the 'PsycINFO' database and CAS, and is the official journal of The International Neuropsychiatric Association (INA). The manuscript management system is completely online and includes a very quick and fair peer-review system, which is all easy to use. Visit http://www.dovepress.com/testimonials.php to read real quotes from published authors. 